\title{
GLOBALIZACIÓN, CAMBIOS EN LA ESTRUCTURA DE PODER Y NUEVAS ELITES EMPRESARIALES: UNA MIRADACOMPARADADE URUGUAY
}

\author{
Miguel Serna
}

RESUMEN

El artículo tiene como objetivo principal abordar la problemática de los cambios en la composición de las elites empresariales en el último ciclo de globalización económica neoliberal desde la literatura teórica comparada reciente y luego un análisis específico a partir de los estudios existentes en la temática sobre Uruguay. El trabajo desarrolla un análisis comparativo de enfoques y estudios sobre elites económicas que señalan cinco tipos de procesos de cambios en las últimas décadas de hegemonía del ciclo de desarrollo neoliberal. En primer término, procesos de convergencia y divergencia entre las trayectorias de las elites políticas y económicas. En segundo lugar, procesos de especialización funcional y diversificación de grupos dirigentes en las distintas fuentes de poder económico. Tercero, la existencia de mecanismos de reclutamiento y reproducción de las elites económicas que se relacionan con las formas de dominación en la sociedad. Cuarto, la producción nuevos y viejos mecanismos de legitimación del poder económico, que combinan formas de capital económico y simbólico. Por último, la creciente centralidad de las redes globales y modos de transnacionalización del capital en la conformación de los grupos de elites empresariales. El análisis de la literatura y estudios sobre elites empresariales en el Uruguay en las últimas décadas muestra tendencias similares (a escala de una economía y país pequeño) donde se está asistiendo a cambios en las composición de las elites empresariales con una creciente importancia del capital transnacional, y de nuevos elencos ejecutivos que se apoyan más en una forma de control y gestión profesionalizada de las empresas, en redes globales y nuevas formas de capital social. Estas transformaciones contrastan con los perfiles clásicos de empresarios reclutados por medio del capital familiar y prestigio heredado y la pertenencia a gremiales tradicionales y círculos empresariales exclusivos, así como de una canalización colectiva más orgánica de las relaciones con el Estado y la política. El estudio de las elites económicas es una temática clásica que en las últimas dos décadas es una temática que ha regresado al debate académico y público debido a los impactos de los procesos de la globalización económica. En Uruguay la temática adquiere fuerte relevancia debido a que se trata de una economía de escala pequeña, alto desarrollo humano y que en los últimos años ha atravesado múltiples transformaciones en el modelo de desarrollo económico, la creciente presencia de capital y empresas trasnacionales, con la emergencia de nuevos actores empresariales en la escena nacional y en un contexto político de giro a la izquierda.

PALAVRAS-CHAVE: elites empresariales; globalización; poder.

\section{INTRODUCCION ${ }^{1}$}

El estudio de las elites económicas es un tema clásico en la Sociología para comprender la naturaleza, rasgos y consecuencias de la estructura de poder en la sociedad. En las últimas dos décadas es una temática que ha regresado al debate académico y público debido a los impactos de los procesos de la globalización económica.

En este contexto, se plantean múltiples interrogantes sobre nuevas y viejas formas estructuración del poder en la sociedad. Por una parte, varios enfoques señalan la concentración en la distribución de poder

${ }^{1}$ Gracias a los revisores anónimos de la Revista de Sociologia e Política por sus sugerencias en la preparación de este artículo. en las estructuras de propiedad del capital económico y la conformación de sistemas de autoridad y gobierno a nivel mundial. Por otra parte, otros enfoques señalan que en forma paralela, se producen mecanismos de poder anclados en procesos organizacionales y relaciones sociales tanto en el ámbito nacional como subnacional. En este sentido, se trata no sólo de comprender la naturaleza estructural del poder, sino también relacional, de las consecuencias psicológicas y organizacionales y su importancia para comprender las nuevas formas de legitimación del capitalismo contemporáneo.

Las transformaciones del capitalismo organizado que ha concitado creciente atención es un cambio organizacional de separación entre dirección y propiedad de las empresas y las consecuencias en la 
constitución de nuevos elencos dirigentes. El desarrollo de organizaciones empresariales complejas genera nuevas fuentes de poder vinculadas a la gestión empresarial, al tradicional empresario propietario, se suma la figura de la alta gerencia en la cabeza de las empresas. No obstante, a diferencia de las empresas de la primera mitad del siglo XX organizadas en la férrea estructura jerárquica de la organización taylorista del trabajo, las últimas décadas han centrado su desarrollo en innovaciones de nuevas formas de organización flexible, basadas en el funcionamiento y control de redes sociales y digitales.

La reproducción del capitalismo contemporáneo como sistema económico dominante no sólo funciona a partir de las lógicas estructurales y empresas en tanto organizaciones complejas para la obtención de capital, sino también ha producido nuevas formas de legitimación micro en la vida cotidiana de los individuos y la generación de nuevos valores relacionados al mundo de la economía, y el trabajo. Así pues, al decir de algunos autores, emergen nuevos sujetos y clases globales que encarnan estos cambios de comportamientos, actitudes, creencias y valores. Se destacan así consecuencias psicológicas en los individuos que ocupan los cuadros directivos y de alta gerencia que incorporan y legitiman las nuevas éticas de trabajo del capitalismo en estilos de vida cotidiana (en el trabajo y en el consumo). Por tanto, el capital social y cultural aparecen como factores claves en la legitimación del poder de las nuevas elites económicas.

Sin embargo, la emergencia de nuevos elencos dirigentes de empresas y las nuevas formas de poder económico no se legitima mecánicamente en las sociedades, entran en disputa o asociación con viejas formas de reproducción del poder en la sociedad. Los nuevos elencos empresariales, por un lado, desafían las fuentes tradicionales de prestigio social asentadas en clubes sociales exclusivos, gremiales empresariales tradicionales y el peso del patrimonio heredado familiarmente y, por otro lado, proponen nuevos ámbitos de encuentro (foros internacionales), activan redes locales (responsabilidad social empresaria), estilos de vida promovidos a través de canales y medios de comunicación específicos (lugares de residencia en barrios de medio y alto nivel socioeconómico con gran oferta de servicios, inversión en infraestructura tecnológica, patrones de alto consumo, experiencias de formación educativa, laboral y de placer en el exterior) y símbolos de estatus legitimados en circuitos internacionales y globales.

Además, la emergencia de nuevas elites económicas abre nuevas interrogantes sobre su influencia y relación con el campo político.
En Uruguay la temática adquiere fuerte relevancia si se tiene en cuenta que se trata de una economía de escala pequeña y que en los últimos años ha atravesado múltiples transformaciones de "giro ideológico a la izquierda", rotación de las elites políticas en el poder, comienzo del nuevo siglo con una crisis y posterior salida con un nuevo ciclo de alto crecimiento económico e incremento de la inversión externa directa, procesos que desencadenaron emergencia de nuevos actores sociales en la escena nacional, abarcando 300 ministros y parlamentarios del período 1999-2009. En un contexto de circulación de las elites políticas en el gobierno (SERNA et alii, 2012) y transformación productiva es clave comprender los cambios e impactos en la composición de las elites empresariales emergentes.

En este contexto, el artículo tiene como objetivo principal abordar la problemática de los cambios en la composición de las elites empresariales en el último ciclo de globalización económica neoliberal desde la literatura teórica comparada reciente y los estudios existentes en la temática sobre Uruguay.

La hipótesis principal a explorar es que se está asistiendo a cambios en las elites empresariales con una creciente importancia del capital transnacional y de nuevos elencos ejecutivos, las cuales se apoyan más en una forma de control y gestión profesionalizada de las empresas, redes globales y nuevas formas de capital social. Esas elites contrastan con los perfiles tradicionales de empresarios basados en el capital familiar y prestigio heredado y en la administración de gremiales y círculos empresariales exclusivos.

\section{ASCENSO AL PODER DE LAS ELITES EMPRE- SARIALES: DIVERSAS PERSPECTIVAS E HIPÓTESIS}

La propia definición de elite ha recibido múltiples connotaciones en la genealogía y uso histórico que se ha dado al concepto, refiriendo a los individuos que ejercen una posición de gobierno y desempeño de tareas de dirección en estructuras de poder objetivadas en un tiempo y espacio determinado.

Los autores clásicos de las elites (Pareto, Mosca y Michels) fundaron una perspectiva donde las elites tenían un papel central en la organización del orden social. La concepción de las elites estaba fundada en una visión aristocrática de la sociedad. La definición de elites parte de la idea que son un estrato superior de individuos destacados por cualidades o talentos superiores, y por su competencia dirigente. En esta perspectiva las elites se constituyen en sujetos colectivos con fuerte cohesión en tanto minorías con fuerte capacidad de auto-organización e intereses 
comunes orientadas en función de la búsqueda del control monopólico del poder. En contraposición a las elites están las masas inorgánicas, atomizadas e irracionales plausibles de manipulación y control, e incapaces de tomar decisiones colectivas. Esta tesis elitista fundacional centraba su foco en el papel dirigente de las elites en la constitución del orden político de la sociedad y su vínculo con la reproducción del poder en la sociedad. Paradójicamente, este paradigma tenía un sesgo elitista conservador con una concepción jerárquica en momentos de cambio histórico con las primeras experiencias de modernización social y democratización política.

La literatura posterior se ocupó de abordar en profundidad y en diversos contextos cuáles eran las fuentes de poder, los rasgos principales de los grupos dirigentes y el papel que cumplían en una estructura $\mathrm{y}$ contexto determinado.

La expansión de los procesos de industrialización y del desarrollo capitalista organizado en el Siglo XX reorientó los debates sobre las elites hacia la relación entre política y economía, así como a las lógicas propias del poder económico.

De los múltiples enfoques y debates en la literatura consagrada sobre las elites (GRYNSZPAN, 1996), a efectos de este artículo se abordan seis tópicos recurrentes en la academia y de gran pertinencia pública para comprender el papel y rasgos de las elites económicas.

\section{II.1. Las relaciones de convergencia y divergencia entre elites políticas y económicas}

Uno de los temas que más debate ha producido ha sido las relaciones interelites y sus capacidades de dirigir en forma coordinada.

Por una parte, desde la tradición académica norteamericana (RUIZ SANCHEZ, 2009) Floyd Hunter y Wright Mills constituyeron pilares de la tesis de la unidad de las elites dirigentes en el poder.

En la perspectiva de Wright Mills los hombres que ocupan los puestos superiores de decisión de la economía - accionistas y altos ejecutivos de las grandes corporaciones - el directorio político y el estado mayor del ejército componían un grupo social unificado con una movilidad e intercambio fluido horizontal entre el poder político, el militar y económico.

La definición de elite parte de una minoría de individuos que ocupan posiciones de poder decisorio en instituciones claves de la estructura social (WRIGHT MILLS, 1989, p. 336). Sobre la base de esas posiciones estructurales se establecen oportunidades e intereses, así como estilos de vida, educación y tipos de personalidad que van conformando la unidad psicológica y social de los altos círculos. Las afinidades psicológicas y sociales provienen no tanto de su origen sino de los círculos de poder y formas de reclutamiento que define un tipo social. Sin embargo, ello no supone una teleología histórica de la supremacía de las elites, ni la conciencia $\mathrm{y}$ homogeneidad a priori de sus intereses. Por tanto, hay que investigar en cada contexto histórico los vínculos entre tipos de estructuras y papeles que cumplen las minorías (idem, p. 30).

En contraposición a este tesis se plantearon enfoques de la escuela pluralista (RUIZ SANCHEZ, 2009) entre los más destacados las investigaciones y argumentos de Robert Dahl. Los pluralistas no niegan la existencia de elites, sin embargo cuestionan la idea de una elite unificada porque sostienen que lo propio de las poliarquías es la competencia por el gobierno entre múltiples líderes y grupos de intereses con diversas formas de control e influencia. Lo que predomina es una forma elitismo democrático (Bachrach), que supone la existencia de un método (Schumpeter) y reglas para la competencia y elección entre diversas elites. En la visión de los pluralistas en sus múltiples variantes (GRYNSZPAN, 1996, p. 51), un argumento central que esgrimen es que si bien el gobierno es ejercido por una minoría lo propio del régimen democrático estaría por el carácter abierto y plural en el acceso a la elite. Asimismo, señalan la existencia de una multiplicidad de grupos sociales de veto, así como de la influencia de la movilización de los sectores sociales no pertenecientes a la elite que oficiarían de mecanismos de reducción de poder y que imposibilitarían un monopolio unificado de control del poder.

También aparecieron posiciones intermedias como las de William Domhoff y James O'Connor (RUIZ SANCHEZ, 2009, p. 177-178) que combinan análisis de clase e institucional, destacando la influencia de la elite corporativa y financiera en la elite de poder, con múltiples influencias de sus intereses y movilidad de personal hacia posiciones en el ámbito político y de gobierno.

II.2. Especialización funcional y diversificación de distintas fuentes de poder económico y grupos dirigentes

Algunas teorías han buscado evitar entrar en las discusiones sobre los presupuestos de las elites como actores colectivos con capacidad dirigente, ni tampoco en las consecuencias sobre los regímenes y sistemas políticos, para centrarse en una definición de grupos funcionales (BOTTOMORE, 1967) de acuerdo a las 
posiciones jerárquicas que ocupan y la función de poder que cumplen. Este tipo de definición pragmática de las elites posee al menos dos méritos relevantes. Por una parte, se orienta a identificar en forma precisa las diversas instituciones y estructuras de poder, con una descripción clara de criterios objetivos que determinan posiciones y categorías sociales en la cúspide del sistema; y por otra parte, se avanza en establecer distintos grupos de elites diferenciando funciones que cumplen, relaciones entre elites y clases, así como circulación de las elites en los diversos ámbitos de la sociedad.

Un ejemplo que puede citar de este tipo de enfoque es el de François-Charles Mougel (1990) que realizó un estudio interesante sobre las elites y el sistema de poder en Gran Bretaña. Se trata de un estudio que busca describir todos los grupos de elites nacionales según los diversas estructuras y sistemas (políticas, económicas y función social - sistema normativo y de socialización). En cuanto a las elites económicas, las diferencia entre elites propietarias y de la gestión y las elites sindicales. Las elites propietarias y de la gestión las clasifica a su vez en grandes sectores, terratenientes, financieras, industriales y comerciales.

\section{II.3. Las estructuras de dominación en la sociedad y} la reproducción de las elites económicas

Desde otras perspectivas, tanto la tradición marxista como de corrientes más europeas, en especial la escuela francesa, la problemática de las elites dirigentes se relacionó con el papel en las formas y estructuras de dominación económica y social. Por un lado, autores marxistas (como Paul Sweezy y Ralf Miliband) dirigieron múltiples críticas a los enfoques previos señalando que las elites no podían ser entendidas en forma independiente de las posiciones de clase social y en el sistema capitalista. Por otro lado, la escuela sociológica francesa más reciente, a partir de la influencia de la perspectiva de Bourdieu, plantea la importancia de entender cómo los campos de poder producen elites y estas reproducen relaciones de dominación.

Para Bourdieu, la dominación en las sociedades de capitalismo organizado no se ejerce simplemente por una clase o elite dirigente, sino que supone el efecto de grupos e instituciones que acumulan diversas especies de capital y reproducen posiciones de privilegio en el espacio social ("escuelas de poder"), y que están en relación de antagonismo para conservar sus posiciones privilegiadas sobre los agentes dominados. El poder y la dominación en las sociedades contemporáneas se estructura en función de un entramado del capital económico y el capital simbólico en sus diversas modalidades, escolar (educativo), cultural (arte y consumo cultural), social (relacional y estilos de vida), informacional (informático, estadístico), jurídico (estado), etc. a través de multiplicidad de lógicas propias e interconexión conflictiva entre los campos.

En este proceso el autor le da un papel central a las escuelas de poder, como ámbitos espacios de formación, de socialización de cuadros dirigentes y de producción de mecanismos de legitimación que combinan estatus provenientes del campo económico, con títulos del campo escolar.

La dominación no sólo tiene aspectos estructurales sino también relacionales (habitus) de los grupos dominantes mediante la incorporación de estrategias de reestructuración de inversiones y reconversión de los capitales para mantener y ampliar el patrimonio. Ello supone por parte de las elites de estrategias y prácticas de reproducción de las diversas formas de capital (económico, cultural y social): patrones de fecundidad, de parentesco, matrimoniales, de sucesión, inversiones económicas, patrimoniales; educativas en instituciones y títulos; institucionales, mecanismos jurídicos; de inversión en capital social y estilos de vida propios y reglas interpersonales de reconocimiento mutuo (de inclusión, cooptación y exclusión).

El desarrollo de las sociedades capitalistas en el siglo XX produjo una diferenciación entre capital económico y simbólico, y al mismo tiempo, nuevas formas de legitimación por medio del capital cultural y social. En su perspectiva, la burguesía se reproduce con mecanismos sociales análogos a la antigua aristocracia de sangre, pero con un principio de legitimación social diferente, la meritocracia escolar produjo una nueva nobleza de toga (reconocida por los títulos educativos y competencia racional).

Múltiples estudios dentro y fuera de Francia siguieron estos conceptos e hipótesis de trabajo. Un ejemplo pertinente para este artículo, la investigación realizada por Michael Hartmann (2000) que analizó altos ejecutivos de empresas en Alemania y Francia para mostrar la influencia en la selección social de los miembros del habitus de clase (capital social) y la existencia de reclutamiento exclusivo por parte de instituciones educativas específicas que legitiman capital cultural. Otro estudio interesante (HARVEY \& MACLEAN, 2008) es una investigación sobre directores de las principales empresas de Inglaterra y Francia que muestra la relevancia del capital social, entendido como un entramado de redes sociales y culturales, de instituciones educativas, lazos familiares y organización que contribuye a la reproducción de las elites en cada país. 
II.4. Capital económico y simbólico: nuevos y viejos mecanismos de legitimación del poder económico

Las transformaciones del capitalismo tardío en el ciclo de desarrollo de las últimas cuatro décadas han reorientado algunos debates. Por una parte, hacia las consecuencias de los cambios en la propiedad de las empresas y las nuevas formas de gestión empresarial sobre las fuentes de poder del capital económico. Por otra parte, comprender el papel de factores socioculturales (ética capitalista y capital social) sobre las nuevas formas de legitimación del capital en la vida social cotidiana. Ambos aspectos tendrían repercusiones relevantes para conformación de nuevas elites empresarias en el capitalismo contemporáneo.

Bolstanski y Chiapello (2002) señalan que la Ética y la cultura han sido mecanismos relevantes para la reproducción y legitimación del capitalismo como modo de producción. Eles distinguen capitalismo de las empresas y empresarios en tanto agentes responsables de la reproducción de la lógica del capital (accionistas, inversores, directores), sujetos con un compromiso personal en el proceso de acumulación.

A su vez, denominan "espíritu capitalista" (retomando la acepción original de Max Weber) como los elementos éticos morales de adhesión al capitalismo, conjunto de creencias y representaciones que contribuyen a justificar el orden capitalista (regulación moral); un "ethos" orientado a un modo de acción que busca el beneficio y el cálculo instrumental.

En cada ciclo de desarrollo capitalista aparecen y se transforman nuevas formas de espíritu capitalista, de mentalidades y compromisos normativos que contribuyen a la reproducción del régimen económico capitalista. El autor distingue tres grandes ciclos.

(i) El "capitalismo racional" original hasta finales del siglo XIX, que se personifica en la figura del burgués emprendedor individual, cuyos valores se asocian a la asunción de riesgo, espíritu innovador, la capacidad de contabilidad y cálculo económico en la vida cotidiana.

(ii) El "capitalismo organizado", que tiene su período de auge entre 1930 y 1960, traslada el énfasis a la organización, la gran empresa industrial centralizada y burocrática (organización taylorista). En la cúspide emerge la figura del Director que conduce la organización racional del trabajo y la planificación de largo plazo.

(iii) El "nuevo espíritu capitalista" aparece entre 1970 y 1990, tiene como epicentro de desarrollo la ciudad, como locus espacio-temporal del capitalismo, y a la administración empresas como saber privilegiado, que moldea el gusto social y la estética. El nuevo espíritu capitalista tiene como rasgos propios el compromiso de las personas con la producción y los negocios, se presenta como un vínculo positivo entre la aspiración de "seguridad" y bienestar material de las personas y el estímulo a la acumulación de capital. Se trata de un espíritu que orienta la acción con una cara bifronte, de deseo e insaciabilidad del consumo y de disciplinamiento económico.

En claves paralelas Richard Sennet $(2000 ; 2006)$ coincide con la transformación de un nuevo capitalismo y una nueva cultura capitalista. La aceleración de la circulación económica de bienes, servicios y fuerza de trabajo hacia finales final del Siglo $\mathrm{XX}$ tuvo consecuencias en la distribución del poder económico. Por una parte se constata una de transferencia poder de los directores a los accionistas y circulación internacional del capital financiero. Los inversores están preocupados por resultados de corto plazo, se trata de un poder anónimo y omnipresente el "capital impaciente", que genera presión permanente a producir cambios institucionales en las empresas "dinámicas", para desarrollar innovación y flexibilidad a los cambios en el entorno económico. Por otra parte, se expande el desarrollo de nuevas tecnologías de comunicación y fabricación que traslada el centro hacia el control de flujos de información e incorporación tecnología avanzada, produciendo una deslocalización del lugar de trabajo y la reducción de los cuerpos burocráticos intermedios de las empresas.

Se producen pues cambios en la organización del trabajo, orientada globalmente y tecnológicamente compleja. Priman formas modernas flexibles de organizaciones: (i) la reinvención discontinua de las instituciones (reingeniería empresarial, técnicas reducción puestos, mayor control, tareas múltiples); (ii) la especialización flexible producción (productos más variados y más rápido, pos fordismo) y (iii) la concentración sin centralización poder (redes menos jerárquicas).

La nueva arquitectura institucional reduce los niveles jerárquicos, produce precarización del empleo y secuenciación no lineal de empresas lo que permite múltiples funciones y tareas, tiempos de trabajo y contratos de corta duración para obtener resultados rápidos y flexibles. Como consecuencia se produce un mayor control central de las decisiones pero con una menor autoridad institucional. Se convive con una renovación permanente de los equipos dirección de las empresas, menos cuadros intermedios de trabajo autodisciplinado y operaciones fragmentadas en el espacio.

Estos cambios sistémicos y organizacionales 
promueven nuevas figuras empresariales, los "Barones de las tecnología", los "capitalistas del riesgo", y "expertos en reingeniería de empresas"2. Nuevos sujetos para un régimen de producción y trabajo más flexible, que convive con la asunción de riesgos, que supone posibilidad de movimientos en redes amorfas y organizaciones flexibles; el empleo a corto plazo, redes de asistencia más cortas e imprevisibles, la pérdida de controles institucionales sobre el tiempo, el trabajo rutinario y las reglas fijas de la burocracia nacional.

La nueva ética tiene impactos subjetivos en la forma de representación del trabajo y legitimación del capitalismo. La relevancia del prestigio moral del trabajo cambia de significado. De una parte se erosiona la ética protestante, del trabajo autodisciplinado, el pensamiento estratégico y la gratificación diferida. De otra es sustituida por una nueva ética, del trabajo en equipo, en tareas específicas inmediatas (capacidades individuales blandas) y la superficialidad degradante (desorganización del tiempo, ruptura con la rutina y el programa a corto plazo). Las redes interpersonales informales y la sensación de independencia son valorizadas como la clave del éxito laboral.

Como contracara la nueva ética trae consecuencias psicológicas negativas, se pasa del temor a la dependencia, a la preocupación por la pérdida de control de sí mismo y vergüenza de sometimiento a otros. Los individuos conviven con una sensación de desorientación personal de vivir en ambigüedad e incertidumbre, pautada por riesgos pesados y recompensas escasas, fuente de depresión y aprensión (ansiedad por lo que pueda ocurrir). Asimismo, se produce un déficit social en la baja lealtad y compromiso institucional.

\section{II.5. La transnacionalización del capital y de las elites empresariales: las "redes globales"}

Una de las transformaciones en los procesos de globalización contemporánea ha sido la progresiva conformación de clases capitalistas transnacionales (SKLAIR, 2005), entre las cuáles se han identificado cuatro fracciones importantes: (i) la fracción corporativa, aquellos que controlan las grandes corporaciones trasnacionales; (ii) la fracción estatal, los políticos y burócratas globalizados; (iii) la fracción técnica, compuesta por profesionales en redes globalizados y (iv) la fracción del consumo, comerciantes y medios de comunicación responsables

2 Lopez Ruiz(2002) analiza cómo se expresan estas diversas contradicciones en los elencos ejecutivos directivos de las empresas transnacionales. por el marketing y moldear los gustos de consumo.

Teorías contemporáneas de la globalización sostienen que estos procesos traen nuevas clases globales (SASSEN, 2007) que se estructuran a partir del posicionamiento y control estratégica de redes mundiales. En ese sentido, se destacan las posiciones claves en la gestión empresas multinacionales, el comercio intraempresarial y transfronterizo.

Estas nuevas clases globales tienen una posición ambivalente entre los ámbitos nacionales y globales, participando de los procesos desnacionalización parcial. Estas clases se asientan en una variedad redes globales de estructuras económicas, políticas y subjetivas (interpersonales. redes globales). Sassen (idem) distingue entre las clases globales privilegiadas a los profesionales y ejecutivos de empresas transnacionales que comparten pautas de cosmopolitismo cultural, expectativas y orientaciones prácticas hacia búsqueda de rentas económicas; el desarrollo de trabajos en ámbitos transnacionales y de organización en redes globales. Otra clase social privilegiada según la autora está compuesta por redes transnacionales de funcionarios públicos que están vinculados a la gobernabilidad en dominios globales redes inter gubernamentales y transnacionales.

La elite corporativa global se organiza en redes globales fuertemente centralizadas que entrelazan múltiples directorios de grandes corporaciones y organizaciones, individuos a lo lardo del mundo (CARROLL \& CARSON, 2003) que coordinan políticas grupales en ámbitos de gobernanza global y contribuyeron a la construcción de un consenso y hegemonía de las ideas neoliberales. La elite corporativa trasnacional desarrolla sus nexos entre grupos corporativos y financieros en las economías nacionales a través de una progresiva incorporación a redes de engranajes globales, que deben ser estudiadas en sus articulaciones nacionales e inserción en una red jerárquica de organizaciones y agentes globales.

Si bien estos enfoques son todavía emergentes en cuanto a sus evidencias empíricas de la existencia de la clase capitalista trasnacional (STAPLES, 2006) son bastante promisorios en cuanto a la posibilidad de entender e investigar las expansión de redes multinacionales y conexiones entre directorios de empresas transnacionales de diferentes países.

\section{II.6. Las elites en perspectiva histórica y comparada}

En los últimos tiempos se han desarrollado estudios de casos históricos y comparados de elites en diversos contextos históricos y países ${ }^{3}$. Estos estudios llaman la atención sobre la relación entre las elites y el contexto 
histórico social de conformación y desarrollo. Así pues, concentran los esfuerzos en comprender la conformación histórica social de largo plazo de los grupos dirigentes, y abordando en profundidad las relaciones sociales, las redes y capital social de los miembros de las elites empresariales. En función de ello, se ha revitalizado el uso de método proposográfico para la reconstrucción de las trayectorias biográficas de los miembros de las elites.

Varios de estos estudios se han centrado en los impactos de las reformas estructurales neoliberales en la composición de las organizaciones empresariales. Uno de los aspectos que se han analizado es sobre el declive relativo del peso de las sociedades rurales símbolos del prestigio tradicional de la propiedad de la tierra $-y$ la creciente influencia del capital financiero internacional, y el saber experto sobre el campo económico - asociaciones de banco y económicas (HEREDIA, 2003). En estos momentos, hay algunos proyectos en curso en esta línea en Argentina, Brasil y Chile que serán utilizados de referencia para la comparación de resultados posteriores.

\section{ELITES EMPRESARIALES EN EL URUGUAY EN TIEMPOS DE GLOBALIZACIÓN NEOLI- BERAL}

Uno de las referencias clásicas sobre la configuración de la elite dirigente del país y sus transformaciones a partir del agotamiento del modelo batllista en la década del 1960 es el estudio de Carlos Real de Azúa (1969). Real de Azúa aborda el período 1958-1968 como un proceso de circulación de elencos políticos tradicionales, y diferenciación elites con el ascenso elites políticas no partidarias (clases agrarias y económicas, elites burocracia civil y militar) y la reducción de la capacidad política de amortiguación de los conflictos sociales. El autor señala procesos contradictorios, por una parte un proceso de diversificación funcionales y especialización de tareas dirigentes, e internacionalización, por otra parte, la fragmentación de las elites, el debilitamiento de las instituciones y recursos de poder en un contexto de creciente crisis de legitimidad y antesala del quiebre institucional.

En este contexto, el personal político, desarrollado autónomo por sistema de compromiso, participación electoral y fortalecimiento clases medias urbanas profesionales - y rurales, prestigio social fue en declive, por liderazgo carismático y jaqueos

\footnotetext{
3 La expansión de empresas multinacionales fuera de los países centrales hace cada vez más necesario el emprendimiento de investigaciones comparadas en países emergentes de la semiferia o periferia. Un ejemplo reciente Fleury et al (2012).
}

provenientes del poder económico. En este marco, señala la aparición de diversas elites emergentes y contra-elites (religiosa, intelectual y educativa, sindical, agraria, bancario-comercial y industrial).

El análisis apunta a mostrar la creciente diversificación funcional y especialización de tareas dirigentes, así como la influencia de los procesos de internacionalización. Distingue varios componentes en la élite económica; la elite agropecuaria, por su vez, es definida en torno a la concentración y propiedad de tierra y con organizaciones gremiales históricas, como la Asociación y Federación Rural. Luego la elite mercantil, con tradición mercantil portuaria y la bancaria (Asociación de Bancos) con creciente influencia en la órbita de la política económica (especialmente, del Banco Central). Las clases agrariaganadera y comercial se destacan por la continuidad familiar-personal de cuadros sus dirigentes en poder. También identifica a la elite industrial, pero en una posición de mayor debilidad debido al declive de la industrialización sustitutiva y la creciente extranjerización, y que no logro imponer una ideología industrialista. Además, señala la presencia de la elite de los medios de comunicación, aunque en una relación dependiente con la política - prensa - y con el resto de las elites económicas, la bancaria, agraria e industrial.

Estas capas dominantes de la riqueza (las elites agraria, bancario-comercial e industrial) poseen concentración de mando, siendo las más consistentes y fijas. Las elites económicas dominantes tienen como fuerzas unificadoras la educación, el estilo de vida y compartir ideologías comunes. Según el autor, la clase económica alta, posee mecanismos de socialización interna, de lugares escogidos y participación de estilos de vida y consumo comunes (zonas vivienda, lugares veraneo, clubes y centros, viajes, gustos adquisitivos bienes). Asimismo, señalan que los titulares del poder económico buscan reforzar su cohesión mostrando formas de esnobismo social asociadas al prestigio social de la riqueza privada.

En el plano político señaló que poseen una fuerte influencia en capacidad de veto, y que se observaba una creciente cercanía de los grupos empresariales con centros económico-financieros, así como la presencia de EEUU.

Luego de la dictadura, de la transición democrática y con el nuevo impulso de la globalización económica neoliberal con las reformas estructurales de la década de 1990, el tema de los cambios en la estructura de poder económico y los empresarios volvieron a estar en agenda. Un trabajo interesante (BÉRTOLA, RODRIGUEZ \& STOLOVICH, 1987) presentó una descripción pormenorizada de la estructura de 
propiedad del capital económico y su organización. Los autores plantean que los procesos de acumulación capitalista refuerzan la concentración y centralización del capital pero que ya no se da solamente por empresas sino en estructuras más amplias de "grupos económicos", que controlan la gestión y propiedad de grandes empresas. La noción de grupo económico, además de ser una unidad económica de concentración de capital posee algunos aspectos sociológicos relevantes. Los grupos responden a asociaciones supraempresariales cuyos vínculos de cohesión son relaciones de grupos sociales y familiares dentro del ámbito nacional. Otro aspecto de interés, son las relaciones entre los grupos económico nacional y la participación de grupos y empresas transnacionales.

Desde perspectivas sociológicas se abordaron otros aspectos relacionados sobre los tipos de empresarios y desarrollo en el país. Así, una tipología en la materia fue la elaborada por Errandonea y Supervielle (1991) de empresarios rentistas, especuladores e integrados, estos últimos de creciente importancia en el marco de la integración regional por tener una mayor flexibilidad $\mathrm{y}$ vinculación con los mercados internacionales.

Algunos estudios se centraron en la relación entre Estado, sistema político y empresarios (DE SIERRA, 1992) en un momento donde reaparecían algunos vínculos visibles entre personalidades y representantes del mundo empresarial y gobierno.

Otro tipo de enfoques que aparecieron, fueron nuevos y viejos estudios sobre cámaras y gremiales empresariales a inicios de los noventa. Por un lado, haciendo nuevas preguntas, de las actitudes y opiniones de los colectivos empresariales en el contexto de creación del Mercosur y de mayor liberalización económica. Por otro lado, estudios de corte historiográfico (CAETANO, 1992; CUNHA, 1992; LACUESTA, 1992; BERETTA, 1994; 1998), politológico (LANZARO, 1992) o sociológico (BRUERA, 1992; BIRLE et alii, 1994; PIÑEIRO \& CARÁMBULA, 2010), que se interrogaron sobre las características de organización colectiva, y cambios de las gremiales tradicionales (Asociación y Federación Rural, Cámara de Industrias, entre otras).

En la última década, reaparecieron estudios sobre empresarios. Algunos de ellos retoman la investigación de las relaciones entre empresarios como sujetos colectivos, modelos de desarrollo y Estado (ZUBRIGEN, 2006).

También se desarrollan estudios desde la historia social y económica (BERETTA, 1994; 1998; BERTINO, 1994) que se preguntan sobre las características de las empresas y empresarios emblemáticos del desarrollo agropecuario e industrial, así como de la formación de ethos burgués en el siglo pasado.

Otra línea de trabajo es la aplicación en forma anual de una encuesta a elites por parte del Instituto de Ciencia Política de la Faculdad de Ciencias Sociales de Universidad de la República (FCS-Udelar). Dicha encuesta releva actitudes y comportamientos políticos de una muestra por cuotas rotativas a miembros elites políticas, alta burocracia, empresariales y sindicales. La encuesta se centra en opiniones sobre temáticas de la agenda pública y variables típicas de cultura política.

El trabajo de Constanza Moreira (1996) sobre los cambios en la cultura política analiza las opiniones y actitudes de los empresarios, en particular sobre la igualdad y los modelos de desarrollo.

En los últimos años, buena parte de la atención se dirigió a los cambios en la estructura económica. El Informe sobre Desarrollo Humano en el Uruguay (ONU. PNUD, 2008) analiza varias tendencias de transformación productiva. La declinación relativa del peso del agro y la industria reactivación en los últimos años. No obstante, se advierte también una "primarización" del sector industrial, con menor capacidad de valor agregado industrial, y de asociación con cadenas del sector primario de la economía. Asimismo, constata un "incremento" del sector servicios, particularmente en los sectores de las comunicaciones y transporte, del sector no transable inmuebles, y hoteles y restaurantes.

Entre los cambios en la estructura económica del país se destaca también el incremento de la inversión de capital externa directa. Esta inversión externa (BITTENCURT et alii, 2009, p. 20) se dirigió a varios sectores, fundamentalmente el sector agropecuario, las comunicaciones, la intermediación financiera, los hoteles y restaurantes.

En el sector agrario se estima que el alrededor del $30 \%$ de la tierra es extranjera-principalmente capitales brasileños y fondos de inversión financiera -, y la inversión externa productiva es particularmente significativa en sectores como el complejo forestal, cerealero - el boom de la soja -, algunas en el sector lácteo y en partes del sector cárnico -frigoríficos (MURIALDO, 2009).

Una parte de la inversión extranjera fue promovida por incentivos y políticas específicas (BITTENCURT et alii, 2009, p. 21) orientada hacia la industria manufacturera - especialmente de forma intensiva en recursos naturales; el comercio y los servicios electricidad, gas y agua; los servicios logísticos y portuarios y las zonas francas. 
Los cambios registrados en los últimos años no sólo son en la propiedad de las empresas, sino también en los modelos organizativos y de gestión de las mismas (ERREA et alii, 2011). La noción de complejos agroindustriales, es enriquecida por modelos de organización empresarial para captar la naturaleza de los cambios. Dentro de los cambios organizativos uno de los elementos recurrentes señalados en la literatura es el papel de las estrategias empresariales y la innovación (MASSERA, 2003).

Estudios sociológicos recientes analizan los cambios de las empresas, empresarios y redes sociales. En el estudio del sector forestal de Piñeiro y Carámbula (2010) llaman la atención en los cómo las nuevas empresas forestales generan nuevas redes locales a través de intervención de responsabilidad social empresaria, sustituyendo el prestigio tradicional local de los empresarios ganaderos. Otra investigación realizada por Rosario Radakovich (2011) muestra que los profesionales y altos ejecutivos poseen altos niveles de capital cultural y tienen patrones específicos de consumo cultural altamente internacionalizados. Por su parte Alfredo Falero (2011) focaliza un estudio de formación de nuevos empresarios con un tipo de capital social y localización espacial determ e caso de una zona franca para mostrar la influencia de procesos de globalización económica y la inada.

\section{CONCLUSIONES: ALGUNAS HIPÓTESIS A MODO DE CIERRE}

El debate teórico contemporáneo destaca varias dimensiones e hipótesis sobre la transformación en los elencos dirigentes del poder económico y sus consecuencias sociales.

Por un lado, una propiedad de empresas más concentrada y trasnacionalizada, y con un tipo de capital financiero que circula rápido e "impaciente" en sus resultados y ávido en la apropiación de la riqueza. En forma paralela, a la creciente importancia de un tipo de capital más anónimo en la naturaleza de la inversión y la titularidad de la acciones, y globalizado en sus alcances económicos, se producen cambios en la cúpula dirigente de las empresas. Una cúpula dirigente de nuevo tipo, que tiene más herramientas de gestión y mecanismos de control de las empresas, pero con una estructura de organizativa flexible, que da una autoridad menos firme, tanto en la relación directorios-alta gerencia y accionista, así como en la relación con los mandos medios y trabajadores.

Por otro lado, la emergencia de nuevas elites dirigentes se enfrenta a la conversión y reconversión con las elites empresariales clásicas, dando lugar a diferentes composiciones en las elites.

La hipótesis general es la creciente importancia del capital transnacional y de las redes globales, que sustituye en parte a los grupos empresariales nacionales que se basan en el capital familiar y patrimonial heredado y el capital social del prestigio acumulado y las gremiales de empresarios tradicionales. Nuevas elites que vienen apoyadas en un modo de profesionalización de la gestión empresarial, visiones, actitudes y estilos de vida asociados a la nueva fase de globalización. En este sentido, es clave estudiar y comprender los cambios en los círculos sociales exclusivos de encuentro, lugares de formación y socialización, así como las nuevas formas relacionamiento con las elites de los poderes públicos. Entre esos dos tipos de empresario, emerge un tercero intermedio, los directivos de empresas públicas, que construyen también sus trayectorias profesional en las redes globales, y entre ámbitos públicos y privados.

En el Uruguay, con sus dimensiones de economía de país pequeño y alto desarrollo humano, varios estudios dan cuenta que las elites empresariales están atravesando varios de esos procesos en los últimos tiempos.

Miguel Serna (miguel@fcs.edu.uy) é Doutor em Ciência Política pela Universidade Federal do Rio Grande do Sul (UFRGS) e Professor de Ciências Sociais e Ciências Econômicas da Universidad de la República (Uruguai).

\section{BIBLIOGRAFÍA}

BERETTA, C.A. 1994. Gremiales empresariales del agro e integración regional: una perspectiva histórica, 1945-1960. Encuentros, Montevideo, n. 3, p. 41-55, abr.

. 1998. Empresarios y gremiales de la industria: asomándonos a medio siglo de historia: de la Liga
Industrial a la Unión Industrial Uruguaya (18791928). Montevideo: Cámara de Industrias del Uruguay.

BÉRTOLA, L.; RODRIGUEZ J. M. \& STOLOVICH, L. 1987. El poder económico en el Uruguay. Montevideo: CUI. 
BIRLE, P.; BRUERA, S.; IMBUSCH, P.; PIÑEIRO, D. E.; RIELLA, A. \& WAGNER, C. 1994. Dos estudios sobre los empresarios y la integración regional. Montevideo: EPPAL.

BITTENCOURT, G.; CARRACELAS, G.; DONESCHI, A. \& LORENZI, N. R. 2009. Tendencias recientes de la inversión extranjera directa en Uruguay. Montevideo: UDELAR. Disponível em: http://www3.bcu.gub.uy/autoriza/ peiees/jor/2009/iees03j3421009.pdf. Acesso em: 8.jan.2013.

BOLTANSKI, L. \& CHIAPELLO, E. 2002. El nuevo espíritu del capitalismo. Madrid: Akal.

BOTTOMORE, T. 1967. Elites and Society. New York: Routlege.

BRUERA, S. 1992. La cámara y los empresarios de la industria: encuentros y desencuentros ante la integración. In: CIESU. (coord.). Organizaciones empresariales y políticas públicas. Montevideo: Trilce.

CAetano, G. 1992. Partidos, estado y cámaras empresariales en el Uruguay contemporáneo: 19001991. In: CIESU. (coord.). Organizaciones empresariales y políticas públicas. Montevideo: Trilce.

CARROLL, W. \& CARSON, C. 2003. The Network of Global Corporations and Elite Policy Groups: a Structure for Transnational Capitalist Class Formation? Global Networks, Hoboken, v. 3, n. 1, p. 29-57, Jan.

CUNHA, N. 1992. Federación rural: gremiales empresariales. Textos auxiliares para el estudio de la historia, Montevideo, Faculdad de Humanidades y Ciencias de la Educación, n. 4.

DE SIERRA, G. 1988. Estado y empresarios en el Uruguay postdictadura. In: GARRIDO, C. (coord.). Empresarios y Estado en América Latina. Ciudad del México: Clacso.

ERRANDONEA, A. \& SUPERVIELLE, M. 2001. Tipos de empresarios, agroindustrialización y Mercosur: reflexiones sobre un desafío inminente. Revista de Ciencias Sociales, Montevideo, n. 6, set.

ERREA, E.; PEYROU, J.; SECCO, J. \& SOUTO, G. 2011. Transformaciones en el agro uruguayo: nuevas instituciones y modelos de organización empresarial. Montevideo: Universidad Católica del Uruguay.

FALERO, A. 2011. Los énclaves informacionales de la periferia capitalista: el caso de Zonaamérica en Uruguay. Un enfoque desde la sociología.
Montevideo: UDELAR.

GRYNSZPAN, M. 1996. A teoría das Elites e sua genealogia consagrada. $B I B$, São Paulo, n. 41, p. 35-83.

HARTMANN, M. 2000. Class-specific Habitus and the Social Reproduction of the Business Elites in Germany and France. The Sociological Review, Hoboken, v. 48, n. 2, p. 262-282, May.

HARVEY, C. \& MACLEAN, M. 2008. Capital Theory and the Dynamics of Elite Business Networks in Britain and France. The Sociological Review, Hoboken, v. 56, n. suplementar s1, p. 103-120, May.

HEREDIA, M. 2003. Reformas estructurales y renovación de las élites económicas en Argentina: estudio de los portavoces de la tierra y del capital. Revista Mexicana de Sociología, Ciudad del México, v. 65, n. 1, p. 67-115, ene--mar. Disponível em: http://www.ejournal.unam.mx/rms/ 2003-1/RMS03103.pdf. Acesso em: 8.jan.2013.

LACUESTA, M. 1992. Camara mercantil: gremiales empresariales. Montevideo: FHCE.

LANZARO, J. 1992. Las cámaras empresariales en el sistema político uruguayo: acciones informales e inscripciones corporativas. In: CIESU. (coord.). Organizaciones empresariales y políticas públicas. Montevideo: Trilce.

MASSERA, E. J. 2003. Estrategias empresariales de conocimiento y desarrollo productivo. In:

El Uruguay desde la Sociología. Montevideo: Universidad de la República.

MOREIRA, C. 1996. La construcción de la agenda pública en Uruguay: una visión desde las elites. In: MALLO, S. (coord.). Ciudadanía y democracia en el Cono Sur. Montevideo: Trazas.

MOUGEL, F.-C. 1990. Elites et systeme de pouvoir en Grande-Bretagne 1945-1987. Bordeaux: Université de Bordeaux.

MURIALDO, M. I. 2009. La incidencia de la inversión extranjera en la industria cárnica. Montevideo: UCUDAL.

PIÑEIRO, D. \& CARÁMBULA, M. 2010. Impactos locales de una industria global: el caso de la agroindustria forestal en el Uruguay. No prelo.

RADAKOVICH, R. 2011. Retrato cultural. Montevideo entre cumbias, tambores y óperas. Montevideo: UDELAR.

REAL DE AZÚA, C. 1969. La clase dirigente. Nuestra Tierra, Montevideo, n. 34, p. 1-60. Disponível em: 
http://www.archivodeprensa.edu.uy/biblioteca/ carlos_real_de_azua/textos/bibliografia/ laclasedirigente.pdf. Acesso em: 8.jan.2013.

RUIZ SÁNCHEZ, J. 2009. Teoría política norteamericana sobre las elites. Su vigencia en el contexto político y económico actual. Espacios Públicos, Ciudad del México, v. 12, n. 26, dic.

SASSEN, S. 2007. La sociología de la globalización. Buenos Aires: Katz.

SENNET, R. 2000. La corrosión del carácter. Barcelona: Anagrama

2006. La cultura del nuevo capitalismo. Barcelona: Anagrama.

SERNA, M.; BOTTINELLI, E.; MANEIRO, C. \& PEREZ, L. 2012. Giro a la izquierda y nuevas elites en Uruguay: ¿renovación o reconversión? Montevideo: CSIC. No prelo.
SKLAIR, L. 2005. The Transnational Capitalist Class and Contemporary Architecture in Globalizing Cities. International Journal of Urban and Regional Research, New York, v. 29, n. 3, p. 485-500, Sep.

STAPLES, C. 2006. Board Interlocks and the Study of the Transnational Capitalist Class. Journal of World-Systems Research, Washington (DC), v. 12, n. 2, p. 309-319, Dec. Disponível em: http:// jwsr.ucr.edu/archive/vol12/number2/pdf/jwsrv12n2-staples.pdf. Acesso em: 7.jan.2013.

WRIGHT MILLS, C. 1987. La elite del poder. Ciudad del México: Fondo de Cultura Económica.

ZUBRIGGEN, C. 2006. Estado, empresarios y redes rentistas durante el proceso sustitutivo de importaciones: los condicionantes históricos de las reformas actuales Montevideo: Banda Oriental.

\section{OUTRAS FONTES}

ONU. PNUD. 2008. Desarrollo Humano en Uruguay. Política, Políticas y Desarrollo Humano. Monte- video: Programa de las Naciones Unidas para el Desarrollo. 


\section{GLOBALIZATION AND STRUCTURE OF POWER CHANGES: NEW URUGUAYAN BUSINESS ELITE IN COMPARATIVE PERSPECTIVE}

\section{Miguel Serna}

The paper wondering about trends and challenges: Which are the main causes of challenges and reproduction of business elites? What are the patterns of Latin-American business elites? What are the consequences on economical recruitment because of neoliberal economic globalization? Which are the main theoretical and comparative explanations? The comparative method identifies five types of elite changes in the last decades of hegemony of neoliberal development cycle. First, convergence and divergence processes between political and economic elite trajectories. Second, functional specialization and diversification processes between elites groups according to different sources of economic power. Third, traditional domination mechanisms reinforce economic elites' reproduction. Fourth, the role of economic and symbolic capital in order to legitimate the economic power. Finally, global networks and modes of capital trans nationalization became more and more relevant on shaping business elites groups. The comparative perspective of the literature and studies on business elites in Uruguay in the last decades shows similar trends (in a small country economy) and changes in the composition of business elites. The growing importance of transnational capital and new managers bases on control and professional management companies, global networks and new forms of social capital. These changes contrast with classic profiles of entrepreneurs recruited through family capital, inherited prestige and traditional union business membership. Moreover, the decline of national union business as main organizations of business interests in front of political power. Economic globalization increased public and academic interest on the study of economic elites. The increasing power of capital and transnational 
corporations in Latin-American countries had several consequences. Uruguay, a small-scale economy, high human development had many transformations in the economic development model in the last decades with the emergence of new business actors on the national scene and in left turn over government.

KEYWORDS: Business Elites; globalization; power. 